\title{
A quadricuspid aortic valve
}

\author{
Erwan Flecher MD, Hervé Corbineau MD PhD, Alain Leguerrier MD
}

\begin{abstract}
A
symptomatic 61-year-old man with a prior history of right coronary disease, which required angioplasty of the middle portion of the right coronary artery was referred to the Pontchaillou Hospital (Rennes, Ile et Vilaine, France) institution for aortic valve replacement. Transthoracic echocardiography confirmed the existence of a central moderate aortic regurgitation (grade 2 ) associated with a severe aortic stenosis (surface area $0.7 \mathrm{~cm}^{2}$, mean gradient $80 \mathrm{mmHg}$ ). The aortic valve appeared bicuspid and the left ventricular (LV) function
\end{abstract}

\begin{abstract}
was normal without LV dilation. The preoperative coronary angiography demonstrated no restenosis of the right coronary artery. The patient underwent openheart surgery, which consisted of an aortic valve replacement by a porcine bioprosthesis. The native aortic valve was excised and found to be quadricuspid, without the usual Y-aspect closure, and with highly calcified and thickened sigmoid cusps (Figure 1). There were no postoperative complications and the patient was discharged eight days after surgery without any other issue.
\end{abstract}

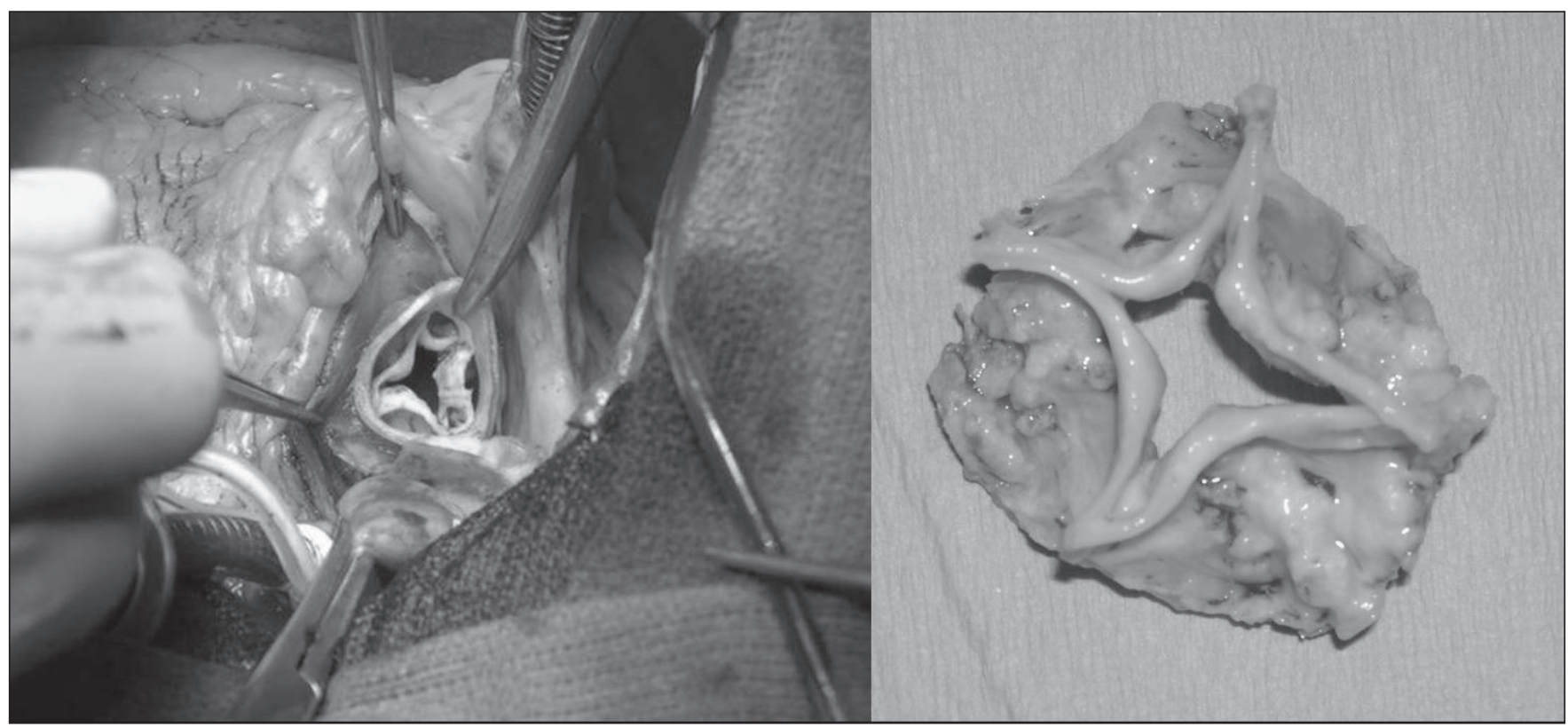

Figure 1) The present patient underwent openheart surgery, which consisted of an aortic valve replacement by a porcine bioprosthesis (left panel). The native aortic valve was excised and found to be quadricuspid, without the usual Y-aspect closure, and with highly calcified and thickened sigmoid cusps (right panel)

Department of Cardiothoracic and vascular surgery, Pontchaillou Hospital, Rennes, Ile et Vilaine, France

Correspondence: Dr Erwan Flecher, Service de Chirurgie Cardio-Thoracique et Vasculaire, Hôpital Pontchaillou, 2 rue Henri Le Guilloux, 35033

Rennes Cedex 09, Ile et Vilaine, France. Telephone 33-2-99-28-24-97, fax 33-2-99-28-24-96, e-mail erwan.flecher@chu-rennes.fr

Received for publication March 30, 2007. Accepted April 8, 2007 